\title{
Il Concetto della Giustizia Secondo l'Alligatore di Massimo Carlotto
}

\author{
Lamiaa Aly Mosselhy Mohamed ${ }^{1}$
}

\begin{abstract}
Through the hard-boiled work of Massimo Carlotto, the research verifies the concept of justice according to his protagonist, the Alligator, an ex-musician, ex-convict and a new private detective. This character discovers a conspiracy well managed years ago in order to save the real guilty person, Mugwump's son, from a murder and send to prison another one instead of him. This new fact (motivates/ urges/ forces) to him decide to declare the (victim's/ imprisoned man's) innocence and restore his dignity back to him. The power of a corrupted lobby and his past as an ex-prisoner prevent him to present evidence, not always collected by legal means, to justice, so he decides to achieve justice by his own and innovative tools in addition to using his own distinguished skills. In the introduction, I elaborated the reasons of choosing a modern writer like Carlotto. The first chapter is dedicated to the characteristics of this gender of mystery work and I provided examples through the writer's work. In the second chapter, I underlined the skills and the modality which reflect the concept of justice adopted by the writer. Finally in the third chapter, I discussed about Carlotto's style.
\end{abstract}

Keywords.Carlotto; Alligatore; giustizia; stampa; hard boiled; delitti.

\section{Introduzione}

Pare che il giallo sia il tipico genere dell'epoca in cui prevalgono azioni politiche cariche di tensioni, guerre, interessi economici, corruzione dei regimi e degli individui, rapine, vittime, l'intervento della polizia e le investigazioni interminabili e la scomparsa della verità in mezzo ai vari mass media, i quali rappresentano partite, politiche o punti di vista soggettivi.

La violenza, l'omicidio e la morte sono diventati elementi della quotidianità. Tra le analisi degli esperti nei telegiornali, le aspettative degli inviati ai combattenti, i rapporti dei corrispondenti, le minacce scambiate tra i vertici delle grandi potenze e l'immagine sanguinosa che proiettano gli schermi spariscono le speranze di pace e di giustizia, anzi si impone soltanto la lingua della forza, del caos e dei progetti capitalistici individuali che manipolano a loro favore le vicende di tutto il mondo.

Questo stato caotico ha suscitato il mio interesse per lo studio del giallo. Un genere così ampio che include diversi sottogeneri. Esso è tra i generi che rendono il lettore così attento ed ansioso da rimanere talvolta con i nervi tesi fino all'ultima riga dell'opera o all'ultima scena del film. La parola giallo oggigiorno include tanti sottogeneri, i quali si distinguono per diverse caratteristiche: il romanzo nero, il poliziesco, il mystery, il noir, lo spionistico, il diabolik, il kriminal e il Satanik. 
Il giallo italiano non manca, anzi risale alla fine degli anni venti quando è stata pubblicata la prima serie di libri gialli edita da Mondadori "con la copertina che mostra l'esagono rosso che spicca su un fondo giallo" (Crovi, 2002,p.43). Parlando di Mondadori bisogna ricordare Alberto Tedeschi, al quale fu affidato l'incarico di dirigere la collana famosa de "Il Giallo Mondador" ed ha contribuito al successo di questo genere in Italia. Nella letteratura italiana il giallo si impone, quindi, fortemente attraverso le collezioni di avventure poliziesche, i racconti d'azione e di mistero, $i$ crimini ambientati in America e i detective stranieri e locali come Artuo Jelling, Duca Lamberti, Il Sergente Sarti, Duca Lamberti, Sasà Jovine e l'Alligatore. (Oliva, 2003). Alcuni giallisti focalizzano sulla criminalità legata ad alcune regioni o zone d'Italia come ad esempio la Sicilia e il potere mafioso come Sciascia ne "Il giorno della civetta", opera in cui ha delineato il rapporto tra potere e mafia negli anni sessanta:

Il giallo per stessa ammissione di Sciascia non può avere soluzione, non può sperare in un happy ending con i colpevoli che vengono puniti in maniera esemplare [...] un giallo senza soluzione [...] che può anche inteso come parabola, di fronte alla realtà come nei riguardi della letteratura, dell'impossibilità di esistenza del giallo in un paese come il nostro; in cui di ogni mistero criminale molti conoscono la soluzione, i colpevoli - ma mai soluzione diventa "ufficiale" e mai i colpevoli vengono, come si suol dire, assicurati alla giustizia (Crovi, 2002,pp.77-78)

Altri autori si occupano di registrare cambiamenti sociali, corruzione di potere giudiziario e lo spostamento della criminalità organizzata a diverse parti dell'Italia settentrionale come ad esempio Pinketts, Lucarelli e Carlotto. (Oliva, 2003). Quindi ci sono tanti scrittori italiani di giallo che hanno ambientato le loro opere in diverse città d'Italia sottolineando vezzi e vizi e usando tecniche molteplici per alimentare la suspense e mantenere viva l'attenzione del lettore fino alla fine dell'avventura. Nel corso del ventennio fascista, alcuni scrittori hanno adattato gli avvenimenti in luoghi esotici. De Angelis invece si dedicava alla descrizione della psicoanalisi dei personaggi, indagando cosa si celasse dietro le tentazioni (Crovi, 2002). Salvo Montalbano si considera tra quelli che hanno avuto una grande fama di pubblico e hanno realizzato un grande successo sia nei gialli di Camilleri che nella loro trasposizione televisiva.

Nonostante ciò sono alla cerca di un giallista italiano più recente e che nello stesso tempo abbia sperimentato un nuovo colore di questo genere. Pertanto Massimo Carlotto può suscitare curiosità per due motivi. In primis l'avere un'esperienza con la giustizia come latitante ed imputato e dunque un suo contatto diretto con questo mondo di tenebre. Il secondo risiede nel protagonista, l'Alligatore, che a mio avviso è un personaggio autobiografico. Lo stesso Carlotto afferma il suo caso unico nella sua opera "Il Fuggiasco":

Sono un caso giudiziario, il "caso Carlotto". Per strada, in treno, in aereo, quando qualcuno mi riconosce mi apostrofa con "Ehi, ma lei è il caso Carlotto". Oltre a essere ricordato come il più lungo e controverso caso della giustizia italiana, vengo studiato all'università come caso limite. E irripetibile.(Carlotto, 1994, p.4)

Carlotto ha scelto del resto un nuovo sottogenere, l'hard boiled, per puntare $\mathrm{i}$ riflettori sulla malavita di Padova degli anni Novanta. Infatti nel primo capitolo emergono le caratteristiche di questo genere per poi rapportarsi, nel secondo capitolo, 
con gli ambienti, i personaggi e l'atmosfera locali. Nel terzo capitolo si giunge ad individuare le tecniche narrative carlottiane.

Ci sono, senz'altro, tanti altri scrittori italiani e stranieri che hanno adottato questo stesso genere, tuttavia l'obiettivo della ricerca rimane sempre la prospettiva e i mezzi proposti da professionista di arrivare alla verità e realizzare la giustizia nella realtà, nella quale è tante volte assente o nascosta.

\section{Il Triangolo della Criminalità}

Il termine dell'hard boiled vuol dire l'uovo sodo e duro che indica in modo simbolico il carattere dell'investigatore che non ragiona soltanto in maniera logica per arrivare ad una soluzione di un caso complesso o misterioso, ma si comporta come l'Alligatore, il protagonista di Carlotto, che si lascia coinvolgere dai pericoli, a volte rischia la vita ed esercita spesso mezzi illegali e violenti per mettere in evidenza la vita nelle metropoli, ma da un angolo diverso di quello della malavita (la droga, la prostituzione, la pornografia) (Domaradzka, 2012)

$\mathrm{Ne}$ "La verità dell'Alligatore" Carlotto ci fa conoscere i suoi personaggi in modo graduale attraverso un detenuto semilibero coinvolto in un omicidio. Strada facendo il detective, Marco Buratti, rivela i veri colpevoli e scioglie il nodo del processo. Sembra un giallo usuale, ma lo scrittore paragona tra un gruppo di malavitosi: latitanti, analisti, strateghi e terroristi e tra una rete segreta di personaggi di spicco: avvocati, giudici, uomini d'affari, medici, professori e impiegati altolocati. Quindi tra due categorie il primo macchiato per sempre da una vecchia condanna e l'altro considerato il fior fiore della società. Egli cerca di condannare i veri colpevoli e rivelare le loro intenzioni criminose, il reato commesso non è altro che un mezzo per mostrare l'ingiustizia in una società in cui i pregiudizi e la corruzione mandano in carcere gente innocente e lascia libera altri macchiati di vergogna.

Il terzo aspetto che mi preme sottolineare è invece presente maggiormente (anche se non esclusivamente) nella variante della detective fiction che qui mi interessa, ossia quella che Todorov ha definito come «roman noir» contrapponendola alla forma classica del «roman à énigme» (Todorov 1989), e che ha il suo modello paradigmatico nel genere hard-boiled. Mi riferisco alla componente epica: il detective infatti tende a assumere i tratti e il ruolo di un moderno 'eroe civilizzatore', che combatte per ristabilire ordine e giustizia in un mondo progressivamente invaso dal caos morale e dalla violenza (Micali,, 2016, p.2)

I corrotti che stanno dietro le azioni vengono scoperti dai delinquenti. Il filo conduttore dell'opera è Magagnin il semilibero, colui che ha passato lunghi anni in prigione per un delitto che risulta poi non aver commesso, venendo dunque scarcerato; egli si trova in seguito coinvolto in un altro omicidio, ai danni di giudice, con modalità del tutto analoghe al primo reato. In questo nuovo caso vengono verificate quasi le stesse prove: le sue orme, il coltello con cui è stata uccisa e la vendetta che giustifica la sua intenzione di uccidere la Belli che l'aveva condannato in precedenza.

Tutte le vicende e il luogo dove è stata rinvenuta la vittima sono state progettate per sviare i sospetti dal vero colpevole e puntare il dito contro Magagnin. I poliziotti e le autorità trascurano di proposito i dettagli nella scena del crimine o talvolta non gli 
attribuiscono l'importanza necessaria. D'altra parte l'avvocato del colpevole si presenta incapace sia di provare la sua innocenza, di cui è certo, sia di trovare l'individuo in semi libertà che ha violato i regolamenti di scarcerazione.

L'Alligatore è un detective autodidatta. Cercando Magagnin, si comporta in modo spontaneo, ma anche da esperto della malavita. Tiene in mente dei piccoli dettagli che gli consentono poi a decifrare degli enigmi complicati. Quando ha scoperto il cadavere della giudice accoltellata, egli osservava con attenzione ogni minimo dettaglio. Le lancette del Rolex al suo polso "segnavano le quattro e trentasei o le sedici e trentasei e il datario indicava il 28 giugno" (Carlotto, 1995, p.42). La stampa invece ha menzionato che "sul polso sinistro un orologio marca Rolex con cinturino metallico, in atto fermo sulle ore sette e trentasei o diciannove e trentasei del giorno 28" (Carlotto, 1995, p.125) . Vuol dire che i poliziotti e gli inquirenti sono arrivati tardi e che qualcun altro era arrivato prima per cambiare la posizione delle lancette in modo da attribuire la colpa a Magagnin.

La sua osservazione accurata è stata la chiave che lo portò ad aprire la scatola cinese. Il sospetto trasmesso a Buratti l'ha indotto a frugare nella casa e ad arrivare quindi ai documenti e alle foto conservati in un angolo segreto a casa sua. A questo punto ha cominciato a cercare i personaggi e le informazioni a riguardo. Cominciò con Giusy Testa, che gestiva una rete di prostituzione e che aveva scandalizzato la giudice con cui condivideva le esercitazioni sadomaso.

Questi dettagli aiutano Burati gradualmente ad arrivare alla verità e rendono il lettore fare l'investigatore alla pari perché smaschera una tra le figure di autorità giudiziaria, si rivela la sua faccia vera e cattiva che dietro la carriera sublime, sfoga la sua aggressività in tendenze sadiche sfruttando persone sottomesse al suo potere come nelle carceri, scuole, manicomi, etc.) (Agliardi, 2010-2011)

Grazie alle abitudini e le memorie che manteneva la giudice, Burati scoprì, inoltre, che ella finanziava le sue serate ricattando $i$ suoi amanti in diversi modi vergognosi:

«Lei era brava, aveva fantasia e riusciva a dominare tutto.»

«Anche Alberto Magagnin»

«Sì, lui più di tutti, All'inizio ero contraria che lo portasse a casa, anche perché era un galeotto e un tossicodipendente magari...aveva l'A.i.d.s. Ma Lei mi convinse che sarebbe stato utile e divertente. Piera e io lo chiamavamo "il barboncino". Faceva tutto quello che volevamo.» (Carlotto, 1995, p.277)

Testa volge l'attenzione verso tre elementi e motivi della criminalità: la corruzione, la prostituzione e la droga, tre aspetti cui partecipano i pezzi grossi della società rinunciando ai valori morali di cui dovrebbero essere i difensori. La giudice Belli ricattava Artoni, il perito, per procurarsi i «quattrini» per i suoi piaceri. Questo ultimo invece di dichiarare il suo errore tecnico legato all'autopsia della prima vittima e salvare così Magagnin, l'innocente, ha sfruttato il processo per ottenere i massimi guadagni possibili da parte del difensore di parte civile:

Ero circondato da troppi concorrenti, per fare carriera avevo bisogno di appoggi [...] Ne fui lusingato. Gli espressi il sollievo e la soddisfazione per il sostegno che mi veniva offerto. Il processo andò come doveva andare e da quel momento iniziai a ricevere incarichi sempre più prestigiosi. (Carlotto, 1995,p.355) 
Nell'investigazione di ogni personaggio emergono chiaramente le caratteristiche dell'hard boiled basato sulla violenza. L'opera inizia con un omicidio brutale, due donne massacrate alla stessa maniera a coltellate. Un'atmosfera di piacere caratterizzata da pratiche sessuali violente che degradano gli uomini al livello degli animali. In questo clima morboso non mancano trafficanti di droga e stupefacenti che commettono delitti violenti di estrema gravità al fine di ottenere una sensazione diversa o zittire la loro coscienza. La corruzione investe il corpo giudiziario e la gente in vista, tra politici, professionisti e uomini d'affari. L'interesse è il criterio secondo cui si decidono i destini degli individui:

Un'ambientazione storica precisa e dettagliata è quella che fa da sfondo alla saga hard boiled ideata da Massimo Carlotto. Il suo Alligatore è cresciuto e vive nella giungla d'asfalto della Padova degli anni Novanta [...] una metropoli melmosa e malsana dove la corruzione è diventata legge e la droga moneta di scambio. (Crovi, 2002,p.159)

Alla scoperta di questo mare di rifiuti ha indotto Buratti ad arrivare alla verità e provare l'innocenza di Magagnin anche se era inutile farlo dopo aver perso gran parte della sua vita in carcere e dopo essere morto per una dose eccessiva di cocaina. L'Alligatore ha deciso di stabilire la giustizia a modo proprio per mandare all'inferno una lunga fila di abusati: "Qui si tratta di scoprire la verità e la verità non può danneggiare Magagnin, che è innocente, ma solo il vero colpevole. Le questioni giuridiche poi si aggiustano." (Carlotto, 1995,p.294)

Questo commento rispecchia un'altra caratteristica dell'hard boiled nel senso che rende più chiara l'indole ambigua del nuovo detective; sembra onesto e sincero, e insiste ad arrivare alla verità ad ogni costo. Il ricorrere ad un investigatore privato verifica d'altra parte l'incapacità della legislazione a difendere il cittadino, ci fa dubitare del valore della legge e l'esistenza della giustizia. La polizia insieme alla corruzione di organi giudiziari e sociali raffinati, in tanti casi, svolgono il ruolo dell'antagonista, perciò l'investigatore si trova costretto a esercitare i mezzi dei criminali per raggiungere il proprio obiettivo nobile. (Domaradzka, 2012)

Alligatore, voce dello scrittore, riassume in poche parole il suo atteggiamento e il suo dolore per il fatto che alcuni, come Carlotto o Buratti, possono provare il rimorso e l'amarezza di Magagnin, di un innocente che il potere giudiziario, la negligenza, l'avidità e l'interesse personale hanno serrato in una cella buia per lunghi anni e l'hanno imputato di un nuovo omicidio solo per coprire i loro scheletri nell'armadio che avrebbero portato a puntare le dita verso di loro. Il detective privato non ci tiene soltanto a smascherare i veri colpevoli, ma anche a salvare la reputazione di uomo semplice immune ai mezzi sporchi dell'ingiustizia; per questo egli cerca di difenderlo a modo, non rispettando, spesso i valori, i regolamenti o la legge perché questi mezzi potrebbero, piuttosto impedire la dimostrazione della sua innocenza, di cui è certo. Inoltre l'autore ci mette davanti alle nostre coscienze, davanti ai nostri pregiudizi che risultano spesso essere senza fondamento. Egli ci fa riflettere sul vero effetto della giustizia nelle nostre società e sulla misura in cui condanna i veri responsabili ponendo una domanda senza risposta: Chi è il vero delinquente? È colui che ruba, uccide, terrorizza l'altro o colui che manipola le nostre vite sulla scacchiera del potere, dei capitali e degli interessi. 


\section{I Mezzi e le Competenze dell'Alligatore}

In primis bisogna conoscere bene questo Alligatore. Chi è? Cosa fa? Come ha avuto questo pseudonimo e come ha guadagnato la buona reputazione presso polizia o nell'ambito della giustizia?

L'Alligatore. Una nuova specie di detective che stabilisce un proprio metodo di investigazione e mantiene ancora alcuni valori e criteri per realizzare la giustizia a modo proprio:

Il personaggio dell'Alligatore si allontana dall'immagine del detective onesto, dedito alla ricerca di giustizia e verità di cui è intrisa la nostra tradizione, e non solo. L'immagine del commissario di polizia perbene e moralmente integro, non sarebbe stata adeguata a questo genere di romanzi; era necessario creare un investigatore che fosse più credibile, in grado di raggiungere la verità evitando il concetto di giustizia di cui avvocati, giudici e poliziotti dovrebbero essere i rappresentanti. (Tasca, 2012/2013, PP.45-46)

È una persona imparziale che non stava né «da una parte né dall'altra». Sembra essere esperto poiché in carcere ha imparato a fare da mediatore per conciliare le liti interne che avrebbero potuto arrecare danno anche ai detenuti più pacifici. Questa sua competenza e la fiducia che ha acquisito grazie alla sua tecnica di detective sordomuto lo rende oggetto di stima di avvocati, latitanti e delinquenti. Un ex musicista che la vita tra le persone deviate ha relativamente contaminato ed ha affinato le sue capacità. Lo pseudonimo:

può attingere pure alle sue abitudini o passioni che costituiscono il suo tratto distintivo, come avviene nel caso dell'Alligatore inventato da Massimo Carlotto. Il detective deve il suo appellativo al gruppo musicale in cui suonava al tempo della giovinezza - Old Red Alligators e indica chiaramente la sua inclinazione per la musica blues.(Domaradzka, 2012, p.71)

Le competenze personali non sono sempre sufficienti. Egli aveva bisogno di assistenti, sono definitivamente i suoi mezzi più efficaci, che superano di gran lunga $i$ mezzi usuali dei poliziotti o degli inquirenti. si tratta di ex prigionieri e criminali ancora in attività, che possono trattare con i loro simili e conoscono bene i segreti di ogni covo di criminale. I primi due erano in regime di semilibertà. Sorvegliavano Magagnin alla sua uscita, dunque conoscevano le sue abitudini, le novità e i fornitori di droga.

In tal modo ogni assistente lo guida nel proprio campo di specializzazione, lo spacciatore gli ha consigliato di cercarlo da Carraro, il quale l'ha guidato verso la casa della giudice che sfruttava Magagnin, il semilibero latitante. Quindi l'Alligatore faceva affidamento a un corpo speciale di detectives e sapeva trattare con loro; alcuni lo aiutano in cambio di soldi, altri gli offrono dei servizi gratuiti o gli restituiscono la cortesia di un vecchio favore. Non gli mancava spesso la persona che gli illuminasse la via della ricerca o che riuscisse a interrogare $i$ vari soggetti coinvolti in manovre subdole, sporche $o$ anche violente:

L'intuito mi diceva di andare a bussare alla porta di Bepi Baldan [...] oltre a essere uno spacciatore, disponeva dei mezzi per fornire protezione a tutti quei latitanti che arrivavano da lui con sufficiente grana [...] passava per un tipo coriaceo, poco propenso a sbottonarsi. Insomma, per farlo parlare ci sarebbe stato bisogno di mostrare i muscoli. Era arrivato il momento di fare entrare in scena Beniamino Rossini, meglio 
noto dell'ambiente come il vecchio Rossini, per distinguerlo dai suoi numerosi fratelli.(Carlotto,1995, pp.59-60)

E' un altro personaggio indispensabile per Burati che lo completa, e nello stesso è così necessario per la trama dell'opera e la suspence degli avvenimenti perché è in grado di commettere $i$ reati che non riesce a fare il protagonista. Entrambi i compagni scambiano gli interessi. Sono due modelli di investigatori: Burati che fa il mediatore e le trattative e Rossini che attraverso le minacce, il ricatto, la forza e l'aggressione recupera il piacere (Domaradzka 2012)

Buratti doveva soltanto cercare Magagnin ed è riuscito a trovarlo attraverso la rete di spacciatori; tuttavia solo dopo aver scoperto l'omicidio della giudice ed aver analizzato gli avvenimenti legandoli al primo delitto per cui è stato condannato questo latitante, l'ha pregiudicato anche lui e stava per fare come tutti gli altri:

La bella avvocatessa avrebbe avuto una brutta sorpresa: il suo cliente aveva fatto il bis. Altro che innocente. Appena preso, avrebbe buttato nel cesso la chiave della cella. Una così era da rinchiudere a vita. Ma in manicomio, non in galera. (Carlotto, 1995,p.4849)

Non si tratta solo di un nuovo omicidio, ma delle conseguenze che pagavano i suoi colleghi in semilibertà giudicati individui pericolosi che potrebbero disturbare la sicurezza della società. Il detective va oltre il reato, pensa come un ex galeotto sospettato che qualche volta ha pagato caro "la colpa di un truffatore che non era tornato da un permesso premio e in più aveva inviato al magistrato di sorveglianza una cartolina di saluti dalla svizzera"(Carlotto, 1995, p.49). Anche quando ha affrontato Magagnin in merito di omicidio e lo interrogava, non lo credeva e pensava che avesse vendicato l'ingiustizia uccidendo la giudice della Corte d'Assise:

«Il nome Piera Belli ti dice niente?»

«㐫 morta» rispose con voce piatta.

«Certo L'hai ammazzata tu».

«Non sono stato io»

$[\ldots]$

«Ho capito tagliato corto».

«D'altronde non sono affari miei. Il mio compito è esclusivamente quello di farti parlare con la Foscarini»(Carlotto, 1995, p.89)

Questo atteggiamento crudele di poliziotti, di ufficiali inquirenti e anche di avvocati si trasforma in determinazione e in tentativi insistenti per arrivare alla verità e per risarcire il povero Magagnin dopo 18 anni in galera, il quale è morto isolato senza avere l'opportunità giusta per difendersi.

Indagando sulle informazioni che ha confessato Magagnin, l'Alligatore insieme al suo assistente, non meno abile, sono riusciti a svelare l'animo anomalo della giudice corrotta, la cui carriera prestigiosa celava dietro condotte sessuali scandalose, nonché tossicodipendente e vera delinquente che approfittava tutti per appagare le sue esigenze:

Ho scoperto la sua innocenza indicai il cadavere, e ora voglio dimostrarla ma per fare questo devo dare nome al bastardo che ha fatto fuori la donna e usato il ragazzo come un burattino fino a spingerlo a spararsi in vena tanta eroina da farsi scoppiare il cuore (Carlotto, 1995, p.162) 
Ad ogni nuova fase l'Alligatore mostra una nuova competenza: dopo aver provato l'innocenza di Magagnin, ha pensato a servirsi di un nuovo mezzo, l'opinione pubblica che, da una parte, rappresenta una pressione sulle autorità per volgere le indagini verso nuovi ipotesi e imputati, dall'altra cerca di rivelare i veri colpevoli, i quali accorgendosi di un nuovo interlocutore che minaccia la loro sicurezza, cercheranno nuovi modi per sottrarsi dai sospetti.

L'unico mezzo per pubblicare le prove trovate a casa della giudice era di passarle alla stampa, in particolare ad un giornalista che ha trattato il delitto in modo diverso senza lasciarsi ingannare delle accuse rivolte interamente a Magagnin ponendo domande e sospettando la presenza di altri colpevoli non ancora svelati. Quando mandava del nuovo materiale al giornalista, lo contattava solo telefonicamente e, per evitare le sorveglianze delle linee da parte delle autorità, gli parlava attraverso cellulari clonati fattigli pervenire attraverso "i ragazzi che portano in giro lettere o piccoli pacchi." (Carlotto, 1995, p.248)

Questo detective impiega tutti i mezzi a sua disposizione: non solo quelli illegali, ma anche quelli sono considerati normali e accettabili, operando scelte prudenti e con estrema cautela. Volendo riconoscere i compagni della giudice nella foto e quindi rintracciarli ha semplicemente contattato un fotografo esperto, "serio, bravo e di poche parole" (Carlotoo, 1995, p.225), a scattare foto in bianco e nero dei partecipanti al funerale.

Di tanto in tanto ricorre ad un nuovo assistente del suo gruppo di delinquenti o dei suoi ex-musicisti. Quando cercava la compagna di Belli che condivideva con lei le serate in compagnia delle prostitute, Beniamino l'ha accompagnato da "Maurizio Camardi. Valente sassofonista e vero esperto delle belle donne di Padova."(Carlotto, 1995, pp.264-265)

I soggetti coinvolti sembrano essere i grani dello stesso rosario, ognuno conduce all'altro finché non si arriva al grano più grande, con cui non si possono esercitare i mezzi usuali come la minaccia, la violenza o le lusinghe. Buratti e Rossini, essendo ex galeotti, non sono in grado di presentare $i$ documenti che hanno trovato alle autorità, tuttavia autenticano ogni nuova investigazione attraverso il registratore:

"Dalla tasca interna della giacca di lino estrassi il registratore e glielo mostrai [...] «Ascoltami bene»[...] Se venissimo a sapere che hai riferito a qualcuno la nostra conversazione o cerchi di identificarci, lo facciamo avere alla stampa...e alla magistratura" (Carlotto, 1995, pp.277-278)

Quando hanno decifrato il codice finale del reato, hanno scoperto due persone considerate appartenenti ai ranghi più alti della società: Sartori, difensore della parte civile nel primo processo in cui hanno condannato Magagnin per la morte di Bianchi, e Ventura il marito capitalista di quest'ultima che è riuscito insieme a Sartori ad allontanare suo figlio, il vero colpevole, dai sospetti, mettendo nei guai un altro innocente.

Bisogna ricorrere ad un analista che sia in grado di consigliargli il modo opportuno per incastrare questi grandi vertici padovani. In questa situazione interviene allora "Max la Memoria", il cui nome rende bene l'idea di computer umano, nel quale si può elaborare i dati a disposizione. Il suo lavoro è di far affari, vale a dire far passare le informazioni, soprattutto se coincidono con i suoi "disegni politici" (Carlotto, 1995, p.294). 
Carlotto ci conduce gradualmente al culmine delle sfide cui un detective potrebbe arrivare, il momento in cui il prezzo della verità potrebbe costargli la vita. Lo scrittore mette in risalto i poteri legali e criminali che possiedono queste persone altolocate, e che sarebbero in grado anche di eliminare le figure che stanno mettendo a repentaglio il loro prestigio. Sartori e Ventura:

Entrano a fare parte dei club e associazioni più esclusivi, per citare il più importante, i cavalieri dell'Ordine di Santa Costanza. Con la benedizione e la copertura, penso inconsapevole, di una parte del clero, questa struttura in realtà raccoglie tutti il marcio di questa città- da vecchi arnesi fascisti implicati in Gladio e varie trame nere, a esponenti corrotti del mondo politico, finanziario, giudiziario, militare - ed è a sua volta trasversale ad altre strutture, lobby o logge massoniche anche estere.(Carlotto, 1995, pp.414-415)

Di fronte a questi prestigiosi personaggi della società non rimaneva ai detectives che trattare con loro specialmente, dopo essere riusciti a sciogliere l'enigma della morte di Bianchini e scoprire che il figlio di Ventura è l'omicida, registrando la confessione dell'infermiere che lo accompagnava nella clinica di salute mentale in cui ha rinchiuso il padre dopo il delitto. Le trattative sono la fase delle scelte decisive, la volontà di avere sempre le mani pulite o usare gli stessi mezzi sanguinosi degli avversari al fine di sopravvivere.

Buratti, "l'Alligatore", appartiene alla categoria degli uomini non ancora degradata o contaminata dalla violenza, mentre Rossini svolge la parte del bandito crudele, convinto che non vi siano soluzioni alternative all'uso della violenza. In altre parole l'autore propone due mezzi che si integrano fra di loro per stabilire la giustizia: le trattative e la violenza. Buratti collegava i pezzi del processo e cercava vie di uscita dopo con Sartori e Ventura:

Voglio trattare. Offro il silenzio in cambio del ritiro della vostra squadra di Killer e della garanzia che non tenterete in futuro azioni di questo o altro tipo nei confronto miei e del mio socio. Per Alberto Magagnin voglio l'assoluzione in istruttoria per l’omicidio Belli e la concessione della libertà condizionata.(Carlotto, 1995, p.603)

Mentre l'amante del blues tentava pacificamente di stabilire la verità, Rossini ha deciso di eliminare $\mathrm{i}$ fratelli Caruso, i camorristi, $\mathrm{i}$ quali li rintracciano per portare a compimento la minaccia nei confronti di Sartori e Ventura:

«Non ce la faccio mormorai, rivolto a entrambi.»

«Non erano questi i patti si lamentò il vecchio Rossini»[...]

«Ho le mie regole spiegai a Rossini. Voi avete le vostre e i "regolari" altre ancora. Ho scelto di fare questo mestiere perché mi permette di non stare con nessuna delle due parti. ogni tanto mi capita di dover mediare con la mia coscienza e di adeguarmi ad alcune regole, ma uccidere significherebbe stravolgere tutta la mia vita e sono vecchio per diventare un altro. Mi dispiace ma non posso farlo»[...]

«Zitto!» Gli intimò il vecchio Rossini e poi, rivolto a me: «Marco, questa volta non ti capisco. Ci troviamo in questa situazione per colpa tua e non abbiamo alternative per salvarci. Se fossero stati loro a prenderci, non solo ci avrebbero ammazzato subito ma si sarebbero anche divertiti ... (Carlotto, 1995, pp.658-660)

Lo scrittore fa spesso come dice Highsmith l'onnisciente, ribadisce attraverso i suoi personaggi la presenza della criminalità organizzata fuori i suoi confini usuali. I 
fratelli Caruso segnalano lo spostamento della Camorra al Veneto, soprattutto a Padova. L'Alligatore indirizza, provando l'innocenza di Magagnin, l'attenzione sulle attualità e le nuove reti di delinquenti (Domaradzka, 2012)

Dopo che Rossini si è sbarazzato dei Caruso, Buratti si serve del suo ultimo mezzo da professionista, contattando una sua ex-amica, tecnica del suono, la quale è riuscita a cancellare le voci di tutti i soggetti coinvolti dai nastri registrati per le comunicazioni di tutti i coinvolti. La tecnica non ha cancellato solo le voci, ma anche ha aggiunto l'ultimo tocco di suspense:

... Bisogna colpire la fantasia della gente mescolando sapientemente giuste dosi di morte, mistero, intrigo, sesso, corruzione del potere. Argomenti che fanno aumentare audience e tirature, si determina così una congiuntura positiva tra la necessità economica dei media di vendere le notizie e il desiderio di una parte consistente dell'opinione pubblica di ingozzarsi di scandali. (Carlotto,1995,p.627)

Alla fine dell'avventura e prima di attraversare $i$ confini nazionali verso la Francia, i due soci hanno depositato il cadavere di Magagnin, conservato a lungo nel congelatore, nel giardino della Villa di Sartori, e ponendo tra le mani un'audiocassetta contenente le confessioni di Artoni; essi hanno inoltre informato Galderisi, il fedele giornalista e l'esponente dei media che renderà clamoroso il caso, il quale giunse per primo al teatro, ben preparato dai Buratti e Rossini. "Seguirono subito una serie di lampi di flash" (Carlotto, 1995, p.676)

\section{Tecniche Narrative}

Quando si parla delle tecniche narrative degli scrittori, si cerca di verificare una modalità diversa che contrassegna l'uso peculiare di un particolare modo rispetto a quello di altri scrittori che hanno in comune la stessa tecnica. Vuol dire che l'autobiografismo è una tecnica adotta quasi in modo naturale da tanti autori che dedicano almeno un'opera della loro narrativa alla loro biografia, tuttavia, mi sembra che Carlotto abbia affermato tale tecnica a modo diverso dell'altro perché ci sono tanti segni nelle sue opere che associano la sua identità con il protagonista, l'Alligatore, Marco Buratti.

Questa affermazione è evidente, innanzitutto, dalle sue abitudini di bere Calvados e ascoltare il blues. Nel Fuggiasco carlottiano si riferisce spesso a queste abitudini che lo accompagnavano anche nei momenti più difficili della sua vita da latitante. Esse costituiscono il suo stile di vita caratteristico, di cui egli non può fare a meno: "A chi, volendo scavare più a fondo, mi chiedeva di definire il concetto di latitanza, rispondevo che "la latitanza è come il blues: uno stato dell'anima"" (Carltto, 1994, p. 37)

"Al protagonista devono magari essere attribuiti un talento o uno svantaggio particolari, come una vista molto acuta o un dito finissimo o una paura morbosa delle falene o delle farfalle" ( Hightsmith, 2007, p.89)

La scelta di questo tipo di musica si riflette nelle poesie inserite tra gli avvenimenti della sua indagine. Infatti ne "La verità dell'Alligatore", di tanto in tanto lui evoca una parte di una canzone blues legata alla situazione, ai suoi sentimenti, al suo umore o alla sua memoria. A volte ascolta un brano prima di dormire o riflettendosi. insomma la musica lo accompagna dovunque vada: 
potevo godermi in pace che tra breve mi sarebbero stati consegnati, comprando dischi e bottiglie di calvados d'annata - dall'altro la mia metà blues ritornava a farsi sentire, e tirandomi per la manica, mi chiedevo di non allontanarmi, di continuare a cercare $[. .$.$] La mia mente pesco un blues adatto all'occasione:$

"You doesed your eyes

And neon spun inside your head

Cause it was dark outside

You read your bible

But God never came” (Carlotto, 1995, pp.128-129)

D'altra parte le parti autobiografiche contribuiscono in modo indiretto. Carlotto ha subito una sorte simile a quella di Magagnin, ma non così negativa poiché nonostante l'ingiustizia che ha subito a lungo, che ha accompagnato la sua vita come un mostro inevitabile, Burati è riuscito a provare la sua innocenza. Magagnin, è invece il simbolo dell'ingiustizia assoluta che non consente riscatto.

Lo scrittore, nonostante abbia informato il lettore che il personaggio si è suicidato, anche a causa di un sovradosaggio, ha reso Magagnin una continua scintilla che ha provocato l'Alligatore tanto da sfidare i corrotti e rivendicare la sua dignità o la dignità di ogni innocente vittima della corruzione. Dunque questo povero ragazzo:

Ha diritto ad avere giustizia anche se è morto [...]

«La giustizia che non hai avuto tu, vero?»

«Cosa c'entra?» Sbottai, innervosito dal fatto che Beniamino paragonasse la mia vita a quella di Magagnin. «Anche te eri il colpevole ideale(Carlotto, 1995, pp.163-164)

Pertanto le memorie carlottiane lo inseguono spesso e lui cerca di sfogare il dolore e le preoccupazioni passate attraverso personaggi soggetti allo stesso destino o altri che hanno deciso di superare questa piaga, lottando contro il male con gli stessi suoi mezzi.

Non c'è dubbio che la latitanza gli abbia fatto acquisire nuove competenze, aguzzare l'ingegno, soprattutto nella necessità. Queste abilità emergono nella descrizione carlottiana che non bada a farci immaginare solo i lineamenti o il loro modo di vestirsi, ma mette in risalto l'intuizione di informazioni segreti che corrispondono poi alle stesse aspettative del detective Alligatore:

Mentre si sedeva, ne approfittai per darle un'occhiata. Scarpe viola col tacco a spillo, tailleur giallo che faceva risaltare un'abbronzatura sorprendente visto che eravamo soltanto alla fine di giugno, atteggiamento apparentemente privo dell'arroganza che contraddistingue i giovani leoni del foro. Sui quarantacinque, rotondetta, piccolina, ben fatta e - ci avrei giurato - separata.

La tecnica descrittiva carlottiana è finalizzata anche ai dettagli connessi a tradizioni per cui si distinguono alcuni soggetti come ad esempio i fratelli Caruso:

"Alfredo e Ugo si assomigliavano molto. Bassi e massicci, indossavano camicie sgargianti aperte sul petto, così da mettere in risalto le immancabili collane d'oro, segno di potere e ricchezza nell'ambiente camorrista." (Carlotto, 1995, p.498)

La descrizione viene spesso associata ad un dialogo che caratterizza anche l'opera carlottiana: il genere e la missione dell'Alligatore implicano fare conversazioni che trasmettono diversi toni e intenzioni. Tra ogni domanda e risposta viene inserito un 
commento descrittivo che si riferisce ad uno stato d'animo, alla rivelazione nelle indagini o al carattere dell'interlocutore:

Si udiva solo il suo respiro affannoso. Nonostante l'aria condizionata alcune gocce di sudore iniziarono a rigarle le tempie fin giù sul collo [...]

«Chi è?» Chiesi, indicando l'uomo mascherato.

«Non siete poliziotti, vero?»

«Chi è?» Insistetti

«Siamo disposti a pagare bene le fotografie»

«Chi è» urlai questa volta toccava a me a fare la parte del cattivo (Carlotto, 1995, pp.268-269)

In un giallo non mancano i momenti di massima tensione, perciò di tanto in tanto l'autore dissemina qualche parte descrivendo un piano come quello poliziesco per inseguire qualcuno o come le avventure e le sparatorie tra $i$ bandisti al fine di trasmetterci questa atmosfera di suspense:

Raggiungemmo via Dini e nascondemmo la moto nel cortile di un condominio. Poi a piedi ci avviammo verso quello dove abitavano i due fratelli e ci appostammo in un angolo particolarmente buio del giardino [...] la grande berlina infilò piano il cancello e altrettanto lentamente si diresse verso il parcheggio. Quando ci passò vicino, uscimmo dal nostro nascondiglio e iniziammo a seguirla. Si fermò qualche metro più avanti. Rossini non aspettò che si spegnesse il motore. Si avvicinò alla portiera di sinistra e punto la mitraglietta sul conducente e attraverso il finestrino aperto permette sulla sua gola la canna silenziata. Nel frattempo, mi infilai nell'auto dalla parte del sedile posteriore destro e appoggiai l'arma sulla nuca di chi mi stava davanti. (Carlotto, 1995,pp. 645-647)

In definitiva la descrizione contribuisce ad inquadrare i mezzi attraverso i quali si può stabilire la giustizia ora con le trattative ora con la violenza.

Sfogliando i gialli di Carlotto, ci si rende conto di un uso peculiare del dialetto padovano. Lo scrittore inserisce queste parti mentre interroga alcuni personaggi nell'opera. Può darsi che Carlotto documenta l'identità del delitto negli anni novanta, lui testimonia attraverso un aspetto così locale la corruzione cha ha contaminato una gran parte della comunità, dei suoi concittadini e non si limita solo ad una categoria di bandisti o ex- galeotti. In altre parole a Padova il male potrebbe sembrare bello:

"Allora», mi rivolsi a quest'ultimo, "Ancora quattro minuti e diamo fuoco a tutta la casa». "Ze per via de chea vecia storia dea clinica? Del ricovero del fiolo de uno dei paroni?» Domando l'ex infermiere [...]

«No'l vora miga denunciarme?»

«No. Ci intersessa solo sapere. poi potrai continuare a occuparti delle tue vigne»

Fu allora che si decie a parlare. [...]

Ero l'infermiere del turno di notte al reparto degli agitati... (Carlotto, 1995, pp.559-590)

Questa persona faceva l'infermiere che ha zittito la propria coscienza per andare in pensione presto e vivere in una villetta in mezzo del suo vigneto. Parlava prima in dialetto cercando di evitare una risposta diretta all'Alligatore, ma alle minacce e l'insistenza dei due soci inquirenti ha confessato. Buratti capiva bene il dialetto, ma lo interrogava in italiano. L'altro interlocutore si è reso conto che era inutile fare il furbo e si è rassegnato dicendo tutta la verità in italiano. 
Quindi, da una parte si potrebbe dire che i dialetti sono un altro tentativo di creare un distacco dalla tradizione letteraria e un segno dell'innovazione linguistica da parte degli scrittori di oggi. Dall'altra, invece, l'uso frequente delle espressioni dialettali risulta, come nel caso dei gerghi e forestierismi, da una verosimiglianza espressiva ovvero la voglia di far esprimere i personaggi rispettando la loro provenienza geografica, sociale, storica (Domaradzka, 2012, p.230)

Tutte queste tecniche insieme ad altre di diversi autori attesta la situazione attuale dell'occidente e le preoccupazioni da un futuro dominato da poteri politici, religiosi ed economici, i quali tessono la trama della criminalità. (Perissinotto, 2008, pp.6,7)

\section{Conclusione}

Questa ricerca ci ha fatto conoscere un giallista italiano moderno che è riuscito attraverso il suo protagonista e i nuovi concetti che propone ad affermarsi tra altri scrittori noti di questo genere. Lui documenta un momento storico del suo paesino decaduto suonando l'allarme. L'autore, illustrando tutti i dettagli di una realtà vergognosa, di una città deteriorata, di azioni e reazioni violente e della coscienza umana in crisi, cerca di destare il lettore ponendo tante domande lasciando la risposta aperta.

L'idea proposta da parte di Carlotto riguardante la giustizia e il modo adottato dal suo protagonista ha soddisfatto alcuni, ma alimenta nello stesso tempo le preoccupazioni di altri. L'assenza della giustizia potrebbe condurre a conseguenze disastrose perché al modo praticato dall'Alligatore non mancano i mezzi violenti. Egli è riuscito a condannare $\mathrm{i}$ veri colpevoli, nonostante facesse il detective di valore, si trova costretto ad eliminare altre persone per salvarsi perché non tutti i malavitosi hanno la stessa mentalità e modalità pacifiche come l'Alligatore. In altre parole se non li uccide lo uccidono. Lo scrittore, quindi, pone l'accento su risultati pericolosi probabili se il concetto della giustizia diventa soggettivo se la comunità viene organizzata e controllata secondo valori e leggi individuali. Il tentativo del detective privato anche se sembra nobile, ma potrebbe portare ad una vita più caotica e insopportabile.

L'esperienza carlottiana con le procedure legali e la giustizia ha reso le idee delle sue opere più efficaci perché di tanto in tanto si identificava con il protagonista, le parti autobiografiche riconosciute attraverso il carattere e i comportamenti dell'Alligatore si considerano un'arte matura dello scrittore che lo distingue tra i giallisti italiani. D'altra parte la sua vita di latitante gli ha fornito altre capacità come la descrizione che ci trasmette un'immagine dettagliata dei lineamenti, dei sentimenti intimi di sofferenza, di timore, di preoccupazione, l'atmosfera di suspense e di violenza. Questo periodo della sua vita gli ha anche consentito una vasta categoria di uomini tra buoni e cattivi il che viene riflesso nello schema dei personaggi nell'opera, i quali incarnano attraverso i loro comportamenti i valori umani sublimi e subdoli.

In definitiva Massimo Carlottiano è riuscito ad adattare l'hard boiled per presentare l'attualità caotica di Padova alla fine del novecento conseguente alla crisi morale e quella di giustizia di cui soffre questa comunità. Questo genere scelto dallo scrittore si integra con l'autobiografismo in modo da rendere le idee trattate più vere e chiare. 


\section{References}

Agliardi, N. (2011-2012), Legge pulsionalità, scrittura, L'immagine carcerario nella letteratura francese contemporanea (dottorato di ricerca in letteratura Euroamericane XXIV Ciclo), , Bergamo, Italia, Università degli studi di Bergamo.

Carlotto, M. (1994), il Fuggiasco, Roma, Italia, Edizioni e/o.

Carlotto, M. (1995), La verità dell'Alligatore, Roma, Italia, Edizioni e/o.

Crovi, L.(2002), Tutti i colori del giallo. Il giallo italiano da De Marchi a Scerbanenco a Camilleri, Venezia, Italia, Marsilio Editore.

Domaradzka,A.(2012), Il nuovo noir italiano, Dal moderno al postmoderno?, Ponzan, Polonia, Università Adama Mickiewicza w Poznaniu.

Highsmith, P. (2007), Come si scrive un giallo, Roma, Italia, minimum fax.

Micali, S. (2007), "Parodie dell'hard-boiled nel romanzo postmoderno: Triste, solitario y final di Osvaldo Soriano", Chi ride ultimo. Parodia satira umorismi, Retrived from http://www.betweenjournal.it/

Oliva, C. (2003), Storia sociale del giallo, Lugano, Italia,Todaro Editore S.A.

Perissinotto, A. (2008), La società dell'indagine:riflessioni sopra il successo del poliziesco, Milano, Italia, Bompiani .

Tasca A. (2012/2013), Scrivere in Giallo il Nordest, il percorso letterario di Massimo Carlotto e Fulvio Ervas (tesi di laurea magistrale in Filologia e letteratura italiana), Venezia, Italia, Università di Ca' Foscari. 